\title{
Up-Regulated ATF4 Expression Increases Cell Sensitivity to Apoptosis in Response to Radiation
}

\author{
Ying Zong $^{\mathrm{a}}$ Shijie Feng ${ }^{\mathrm{a}}$ Chenlin Yu ${ }^{\mathrm{b}}$ Jinwei Cheng ${ }^{\mathrm{c}}$ Guocai Lu ${ }^{\mathrm{a}, \mathrm{d}}$ \\ aDepartment of Health Toxicology, Second Military Medical University, 'Laboratory Animal Center, \\ Second Military Medical University, 'Department of Ophthalmology, Shanghai Chang Zheng Hospital, \\ Second Military Medical University, Shanghai, dCTI Biotechnology (Suzhou) Co., Ltd, Jiangsu, P.R. China
}

\section{Key Words}

ATF4 • Radiation $\cdot$ Apoptosis $・$ ROS

\begin{abstract}
Background/Aims: Activating transcription factor 4 (ATF4) is a member of the activating transcription factor family which regulates the expression of genes involved in amino acid metabolism, redox homeostasis and ER stress responses. ATF4 is also over-expressed in human solid tumors, although its effect on responsiveness to radiation is largely unexplored. Methods: Real-time PCR was used to detect ATF4 mRNA levels in cells treated with different doses of ${ }^{60} \mathrm{Co}$ radiation. Cell viability was assayed using a cell counting kit. The cell cycle was analyzed using flow cytometry, and cell apoptosis was assayed using Annexin V-PI double labeling. Small interfering RNA (siRNA) against ATF4 was transfected into ECV304 cells using Lipofectamine 2000. An ATF4 over-expression plasmid (p-ATF4-CGN) was transfected into HEK293 cells that endogenously expressed low levels of ATF4. The levels of intracellular reactive oxygen species (ROS) were measured using CM-H2DCFDA as a probe. Results: ATF4 mRNA and protein expression levels were higher after radiation and increased in a dose- and time-dependent manner in AHH1 lymphoblast cells $(P<0.05)$. An increase in ATF4 levels was also observed after radiation in primary murine spleen cells, human endothelial ECV304 cells, human liver LO2 cells, breast cancer MCF7 cells, and human hepatocellular carcinoma HEPG2 cells. No change was observed in human embryonic kidney 293 (HEK293) cells. Overexpressing ATF4 in HEK293 cells inhibited cell proliferation, increased cell apoptosis and significantly increased the proportion of cells in G1 phase. Conversely, when ATF4 expression was knocked down using siRNA in ECV304 cells, it protected the cells from radiation-induced apoptosis. These findings suggest that ATF4 may play a role in radiation-induced cell killing by inhibiting cell proliferation and promoting cell apoptosis. Conclusions: In this study, we found that radiation up-regulated the expression of ATF4. We used ATF4 knockdown and overexpression systems to show that ATF4 may play a role in radiation-induced cellular apoptosis.
\end{abstract}

Y. Zong and S. Feng contributed equally to this work. 


\section{Introduction}

Activating transcription factor 4 (ATF4), a member of the ATF/CREB family, plays a major role in regulating genes that are involved in the integrated stress response (ISR) [1, 2], amino acid metabolism, redox homeostasis and endoplasmic reticulum (ER) stress responses. Previous studies showed that ATF4 is over-expressed in many human solid tumors, such as lung cancer, prostate cancer and human hepatocellular carcinoma, suggesting that it may play important roles in tumor progression $[3,4]$.

ATF4 is induced by numerous stress signals, including anoxia/hypoxia, ER stress, amino acid deprivation, and oxidative stress. ATF4 expression is regulated transcriptionally and translationally by the PERK pathway, which involves the phosphorylation of eIF2 $\alpha$, and posttranslationally by phosphorylation, which targets ATF4 for proteasomal degradation [5]. Previous studies have demonstrated that ATF4 is involved in oncogenic process. For example, Horiguchi et al. reported that ATF4 promoted oncogene-induced neoplastic transformation by suppressing the expression of the cellular senescence-associated genes INK4a and ARF $[6,7]$. ATF4 over-expressing cell lines were used to show that ATF4 increased drug resistance to cisplatin, doxorubicin, etoposide, SN-38 and vincristine [8]. Li et al. reported that the knockdown of PERK and ATF4 attenuated LPS-induced autophagy and promoted cell survival [9]. ATF4 is the master coordinator of the integrated stress response (ISR), which is an adaptive pathway that is triggered by multiple stressors. Magne et al. observed that treating liver cells with ethanol up-regulated ATF4 as well as the expression of ISR target genes (i.e., HMOX-1, GCLC, AsnS, IGFBP-1, GADD34, CHOP, ATF3, and CHAC1) [10]. Increased CHOP, GADD34, ATF3, and CHAC1 expression has been associated with growth arrest and apoptosis [11]. CHOP controls Puma expression in islet cells that are experiencing ER stress [12]. It has been suggested that CHOP induces cell death via a variety of mechanisms that include depleting cellular glutathione, sensitizing the cell to oxidative stress, up-regulating pro-apoptotic genes [13] and down-regulating the pro-survival molecule Bcl-2 [14]. ER stress, which is induced by the activation of CHOP, has been shown to activate the proapoptotic molecules Bim and Puma in several cell types and tissues.

Ionizing radiation (IR) activates the unfolded protein response (UPR) and modulates important radiosensitivity-associated factors. Kim et al. observed enhanced UPR signaling in cells exposed to IR, and IR induced eIF $2 \alpha$ phosphorylation and increased ATF4 levels in both HUVECs and HCAECs [15]. Irradiation (IR) can directly or indirectly cause damage to biological molecules to affect cell viability. IR-induced DNA damage activated NF- $\kappa B$ in Glioblastoma cells which promoted expression of IL-6, IL-8 and Bcl-xL, thereby contributing to cell survival and invasion [16]. Recent investigations have suggested that in addition to DNA, proteins can be important targets of IR that can also cause damage $[17,18]$. It has also been shown that IR increases the mRNA levels of ATF4 target genes in a dose-dependent manner [15].

However, the precise function of ATF4 in response to ${ }^{60} \mathrm{Co}$ radiation has never been reported. In the present study, we found that ${ }^{60} \mathrm{Co}$ radiation up-regulated ATF4 expression in different types of cells. We also investigated the function of ATF4 in radiation using knockdown and overexpression systems. Thus, the aim of this study was to investigate the role of ATF4 in ${ }^{60} \mathrm{Co}$ radiation and assess whether ATF4 promotes sensitivity to IR.

\section{Materials and Methods}

Cell culture and reagents

AHH1, ECV304, MCF7, LO2, HEPG2 and HEK293 cells were purchased from the Type Culture Collection of the Chinese Academy of Sciences (Shanghai, China) and cultured in recommended growth media (Invitrogen, USA) supplemented with 10\% FCS, 100 units $/ \mathrm{ml}$ penicillin and $100 \mu \mathrm{g} / \mathrm{ml}$ streptomycin. The cells were maintained at $37^{\circ} \mathrm{C}$ in $95 \%$ humidity and $5 \% \mathrm{CO}_{2}$. The ROS DCFH-DA and BRDU $\mathrm{U}^{+}$were purchased from Genmed Scientifics (Shanghai, China). Anti-ATF4 antibodies were purchased from Abcam 


\section{Cellular Physiology Cell Physiol Biochem 2017;41:784-794 \begin{tabular}{l|l|l} 
DOI: 10.1159/000458742 & (C) 2017 S. Karger AG, Basel
\end{tabular}

(Univ. Biomart, Shanghai, China). All siRNA probes were purchased from Sangon Biotechnology (Sangon Biotech Co., Ltd., Shanghai, China).

\section{Primary spleen cell collection}

Spleens were dissected for primary cell collection. The whole spleen was washed three times using $10 \mathrm{~mL}$ of sterile phosphate-buffered saline $(0.85 \% \mathrm{NaCl}$ and $10 \mathrm{mM} \mathrm{Na} 2 \mathrm{HPO} 4 \mathrm{pH} 7.4)$. The spleen was then diced using scissors in $5 \mathrm{~mL}$ of $0.2 \%$ trypsin-EDTA solution and incubated while gently shaking for $30 \mathrm{~min}$ until the tissue dissociated. The dissociation step was stopped by the addition of 4 volumes of complete Eagle's minimal essential medium (EMEM, Sigma, Univ. Biomart, Shanghai, China) supplemented with $25 \mathrm{mM}$ HEPES. The dissociated spleen cells were passed through a $100 \mu \mathrm{m}$ cell strainer to remove undissociated cells and connective tissue and to obtain a homogenous cell suspension. The cell suspension was centrifuged at $200 \mathrm{~g}$ for $10 \mathrm{~min}$ at $25^{\circ} \mathrm{C}$, and the supernatant was then discarded. The cell pellet was washed twice using complete EMEM, and the cell density was adjusted to $5 \times 10^{6}$ cells $/ \mathrm{mL}$ for subsequent experiments [19].

\section{Analysis of apoptosis}

Apoptosis was evaluated using an Annexin V apoptosis kit (BD Pharmingen). Cells were washed with ice-cold PBS and then re-suspended in binding buffer (10 mM HEPES, pH 7.4, $140 \mathrm{mM} \mathrm{NaCl}$, and $2.5 \mathrm{mM}$ $\mathrm{CaCl}_{2}$ ) at a concentration of $1 \times 10^{6}$ cells $/ \mathrm{ml}$. Cells were incubated with $5 \mu \mathrm{l}$ each of annexin V and PI. The samples were then examined using fluorescence-activated cell sorting (FACS), and the results were analyzed using CellQuest software (Becton Dickinson, San Jose, CA), as previously described [20-22].

\section{Transfection}

Cells were cultured and transfected as previously described [20-22]. Briefly, the ATF4 siRNAs (ATF4 KD1: 5'-CUGCUUACGUUGCCAUGAUTT-3', KD2: 5'-CCCUUCAGAUAAUGAUAGUTT-3', and KD3: CCUGAAAGAUUUGAUAUAATT-3') were pooled. These siRNAs were custom synthesized by Sangon Biotechnology (Sangon Biotech Co., Ltd., Shanghai, China). ECV304 cells were transfected with siRNAs (40 nmol/L) using Lipofectamine 2000 (Invitrogen; ON, Canada) [20-22]. PCR-amplified human ATF4 coding sequences were inserted into the expression vector p-CGN. The p-CGN vectors were transfected into HEK293 cells using an Amaxa electroporation apparatus (Amaxa Biosystems, Koln, Germany) as previously described, with minor modifications [10, 20, 23, 24]. The cells were infected $48 \mathrm{~h}$ later and then subjected to further experiments.

RNA extraction and real-time PCR

RNA obtained from different radiation-induced cells was homogenized in an RNASound ${ }^{\mathrm{TM}}$ RNA Extraction Card (Fortius Bio) and isolated according to the manufacturer's instructions. The reverse transcription products were amplified using a SYBR Premix Ex Taq ${ }^{\text {TM }}$ II kit (TaKaRa) using 7500 software V2.0.6 and the following program: $95^{\circ} \mathrm{C}$ for $30 \mathrm{~s}$ followed by 40 cycles at $95^{\circ} \mathrm{C}$ for $5 \mathrm{~s}$ and $60^{\circ} \mathrm{C}$ for 45 s. The following specific primers were used for PCR: ATF4 forward, 5'-CCCCTTCACCTTCTTACAACC-3'; ATF4 reverse, 5'-GGGCTCATACAGATGCCACTA -3'; PUMA forward, 5'-TGTCCTGGATGAGGATGTGA-3'; PUMA reverse, 5'-GCAGTTAGCAGGGGACTGAG-3';CHOP forward, 5'-GCAGGGTGTGAGAGAAGAGG-3'; CHOP reverse, 5'-TGTGCAAAGGCAAACTCTTG-3'; $\beta$-actin forward, 5'-CTCCATCCTGGCCTCGCTGT-3'; and $\beta$-actin reverse, 5'-GCTGTCACCTTCACCGTTCC -3' (Sangon Biotech Co., Ltd., Shanghai, China). Each experiment was run in triplicate and repeated at least three times. Gene expression was calculated using the $2^{-\Delta \Delta C t}$ method [17], and $\beta$-actin expression was used as a reference value. An ABI 7500 Real-Time PCR Detection System with the Applied Biosystems 7500 SDS Software V2.0.6 was used for these experiments [11].

\section{Cell proliferation}

Cell proliferation was evaluated using MTT assays as previously described [20]. Briefly, after cells were transfected at $37^{\circ} \mathrm{C}$ in $5 \% \mathrm{CO}_{2}$ for different time periods, we gently removed any unattached cells using HANKS buffer. Then, $100 \mu \mathrm{L}$ of DMEM and $20 \mu \mathrm{L}$ of MTT ( $5 \mathrm{mg} / \mathrm{mL}$ ) (Sigma, USA) were added to each well. After the cells were incubated at $37^{\circ} \mathrm{C}$ for $4 \mathrm{~h}$, we discarded the medium and added $200 \mu \mathrm{L}$ of $0.04 \mathrm{M}$ hydrochloric acid in isopropanol to each well. The amount of MTT formazan product in each well was determined by measuring absorbance using a microplate reader at a test wavelength of $490 \mathrm{~nm}$ and a reference wavelength of $630 \mathrm{~nm}$. Each assay was performed in triplicate. 
Cell cycle analysis

Cells were transfected using p-CGN or p-ATF4-CGN, which were synthetized by Sangon Biotech Co., Ltd. (Shanghai, China), for $48 \mathrm{~h}$ and then harvested using trypsin. Harvested cells were washed, resuspended in PBS supplemented with 1\% FBS, and fixed with cold ethanol. The fixed cells were then incubated in phosphate-citric acid buffer for 5 minutes and re-suspended in PI/RNase solution (PBS, RNase, and 50 $\mathrm{mg} / \mathrm{ml} \mathrm{PI}$ ) for the staining step. The cells were incubated in this solution for 15 minutes at $37^{\circ} \mathrm{C}$ in the dark and then analyzed using flow cytometry using a BD FACSCalibur (BD Biosciences, San Jose, CA) and FlowJo software (Tree Star, Inc., Ashland, OR).

\section{Western blot}

HEK293 cells were incubated in serum-free medium with p-CGN or p-ATF4-CGN for $48 \mathrm{~h}$. The lysates from the HEK293 cells were prepared in RIPA buffer containing protease and phosphatase inhibitors. An equal volume $(30 \mu \mathrm{L})$ of each sample was subjected to SDS-PAGE. The resolved samples were transferred onto $0.45 \mu \mathrm{m}$ PVDF membranes and blocked for $1 \mathrm{~h}$ in $5 \%$ milk. They were then incubated overnight at $4^{\circ} \mathrm{C}$ with primary antibodies (anti-ATF4, R\&D, Catalog \#MAB7218, 1:250), and then secondary antibodies (antiMouse IgG secondary antibodies, catalog \#HAF007) at room temperature for $1 \mathrm{~h}$. Anti-PUMA, anti-CHOP antibodies were purchased from Abcam (Trading Company Ltd, Shanghai, China),

\section{Assay for intracellular ROS}

Fluorescence spectrophotometry was used to measure the production of intracellular ROS. CMH2DCFDA (Invitrogen) was used as the probe. HEK293 cells were infected with p-CGN or p-ATF4-CGN for $48 \mathrm{~h}$. The cells were grown to $90 \%$ confluence, washed with warm DPBS and then harvested using trypsinization ( $250 \mu \mathrm{l}$ of trypsin per well), according to the protocol. The cells were then washed four times and incubated with $10 \mu \mathrm{M} \mathrm{CM}-\mathrm{H} 2 \mathrm{DCFDA}$ for $45 \mathrm{~min}$ at $37^{\circ} \mathrm{C}$ in a relatively high humidity (95\%) atmosphere containing a controlled level of $\mathrm{CO}_{2}(5 \%)$ in the dark. The intensity of fluorescence $(490 \mathrm{~nm}$ excitation and $527 \mathrm{~nm}$ emission) was recorded using a Tecan Genios Microplate Reader (Infinite M200). The obtained values (fluorescence/mg protein) are shown relative to the number of p-CGN infected cells (set to 100\%).

\section{Statistical analysis}

The results are expressed as the mean \pm S.D. and represent at least three experiments that were performed in either duplicate or quadruplicate. Statistical differences were analyzed using paired, twotailed $t$ tests.

\section{Results}

${ }^{60} \mathrm{Co \gamma}$ radiation up-regulates ATF4 expression

AHH1 lymphoblast cells were exposed to different doses $(0,0.5,1,2,4$ and $8 \mathrm{~Gy})$ of ${ }^{60} \mathrm{Co \gamma}$ IR for $4 \mathrm{~h}$. ATF 4 mRNA and protein levels were then examined using RT-PCR and western blot analysis, respectively. We found that 0.5 Gy radiation enhanced the mRNA expression of ATF4 and that maximal induction (approximately 3.8-fold more than the control) was observed at 4 Gy radiation (Fig. 1A). ATF4 protein expression levels were correspondingly increased (Fig. 1C). We next treated cells with 2 Gy IR and recorded data at different time points $(0,4,8,12,24,48$ and $96 \mathrm{~h})$. ATF4 mRNA levels peaked at $4 \mathrm{~h}$ and then gradually declined in a time-dependent manner. However, the level remained higher at $96 \mathrm{~h}$ in the treated cells than in the controls (Fig. 1B). A similar result was observed for ATF4 protein levels (Fig. 1D). We next isolated murine primary spleen cells, and we found that radiation increased ATF4 mRNA expression levels in a dose- and time- dependent manner (Fig. 2). The radiation-induced increase in ATF4 expression was also observed in human umbilical vein endothelial (ECV304), human normal liver (LO2) and some tumor cell lines, including breast cancer (MCF7) and liver cancer (HEPG2) cells, but not human embryonic kidney (HEK293) cells (Fig. 3). These data demonstrate that ${ }^{60} \mathrm{Co} \gamma$ radiation dramatically up-regulates ATF4 expression levels. 


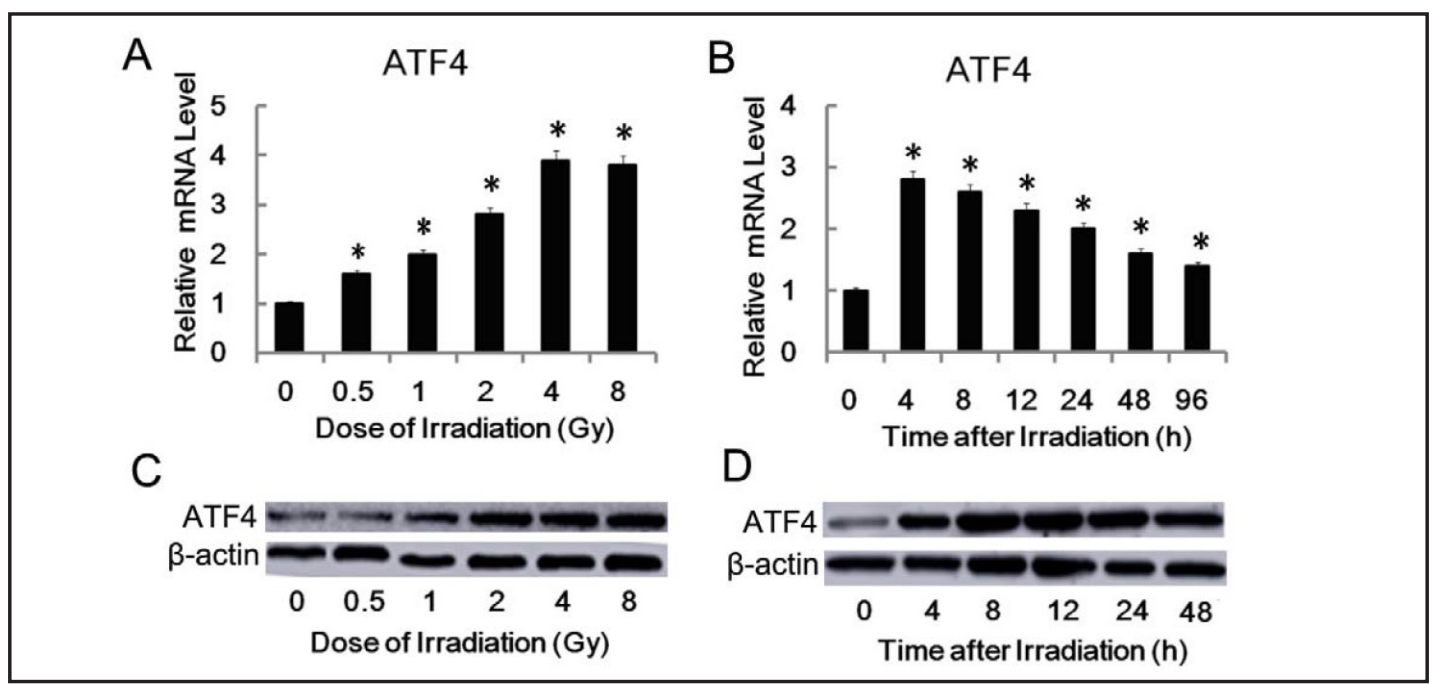

Fig. 1. IR induced ATF4 in a dose \& time dependent manner in AHH1 Cells. The expression of ATF4 in AHH1 Cells was detected using real-time PCR and western blot respectively. The relative mRNA level was up-regulated in a dose-dependent manner in eight hours after irradiation and maximal induction was observed at 4 Gy radiation (approximately 3.8-fold relative to control) (Fig. 1A). Cells were treated with 2Gy irradiation for different time intervals ATF4 mRNA was increased to a peak at 4 hours and gradually declined in a time-dependent manner, but still increased at $96 \mathrm{~h}$ relative to control (Fig. 1B). Results are representative of 3 independent experiments $* \mathrm{P}<0.05$ vs. Control. Expression of ATF4 protein (Fig. 2C\&D) and mRNA are in the same manner after irradiation.

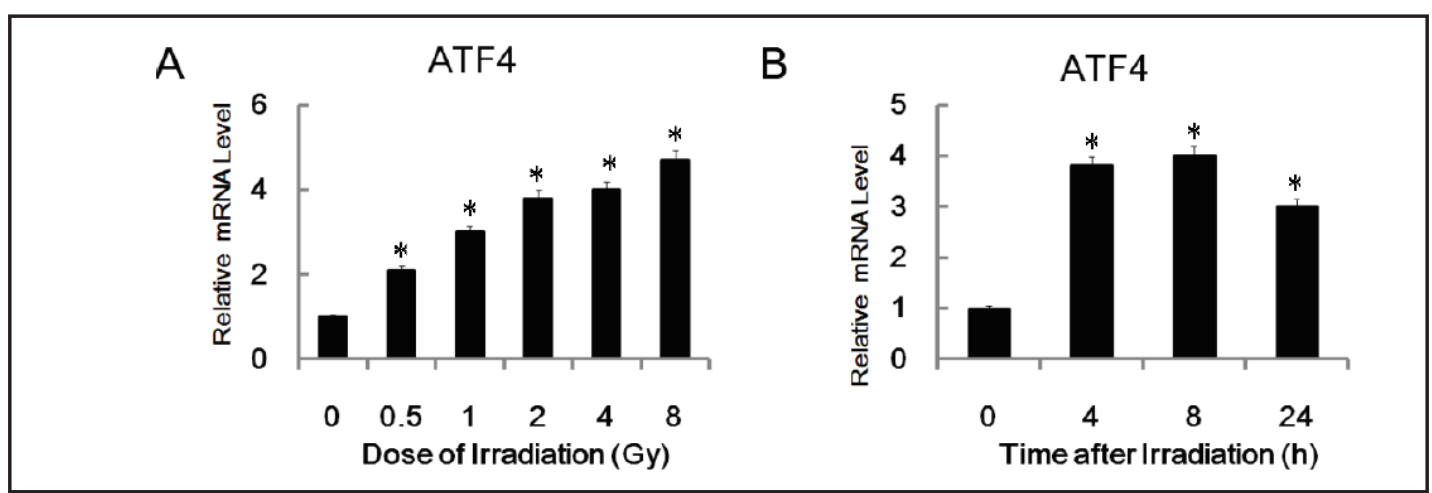

Fig. 2. ATF4 mRNA can be induced by IR in primary murine spleen cells in both a dose \& time dependent manner in vivo. The relative expression of ATF4 mRNA in primary murine spleen cells were detected using real-time PCR. The relative mRNA level was up-regulated in a dose-dependent manner in eight hours after irradiation (Fig. 2A). When cells treated with 2Gy irradiation for different time intervals, ATF4 mRNA was increased to a peak at 8 hours and gradually declined at 24 hours, but still increased at $24 \mathrm{~h}$ relative to control (Fig. 2B). Results are representative of 3 independent experiments $* \mathrm{P}<0.05 \mathrm{vs}$. control.

Over-expressed ATF4 confers anti-proliferative and pro-apoptotic effects

To explore the effect of IR-induced ATF4, we transfected the ATF4 over-expression plasmid p-ATF4-CGN or the control plasmid into HEK293 cells, which express low levels of ATF 4 mRNA. The level of ATF4 ectopic expression was confirmed using both quantitative RTPCR and western blot analysis (Fig. 4A \& B). We counted cell numbers using flow cytometry after different time intervals $(2,4,6$, and 8 days) in ATF4-overexpressing or control HEK293 cells and found that the number of ATF4-overexpressing HEK 293 cells was significantly lower than the number of control cells after 6 and 8 day (Fig. 5A). We next detected cell apoptosis and found that the proportion of apoptotic cells began to be higher after $4 \mathrm{~h}$ in 
Fig. 3. ATF4 mRNA can be induced by IR in several human cell lines. The relative expression of ATF4 mRNA were detected using real-time PCR in ECV304, MCF7, LO2, HEPG2 and HEK293 cell lines .All cells were detected in 4 hours after 2 GY irradiation. Results are representative of 3 independent experiments $* \mathrm{P}<0.05$ vs. control.

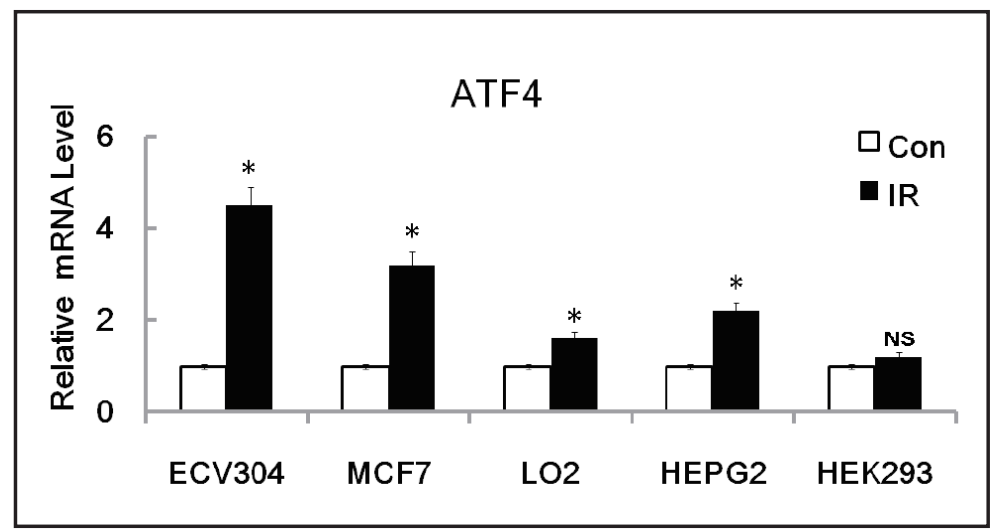

Fig. 4. ATF4 was up-regulated in HEK 293 cells with an ATF4 over-expression system, and successfully down-regulated in ECV304 knockdown cell lines. The relative expression of ATF4 mRNA in HEK 293 cells was detected using real-time PCR (Fig. 4A). Immunoblots to measure protein levels in ATF4 transfected HEK 293 cells (Fig. 4B). Different ATF4 siRNA and non-specific control siRNA (NC KD) were transfected into ECV304 cells using an

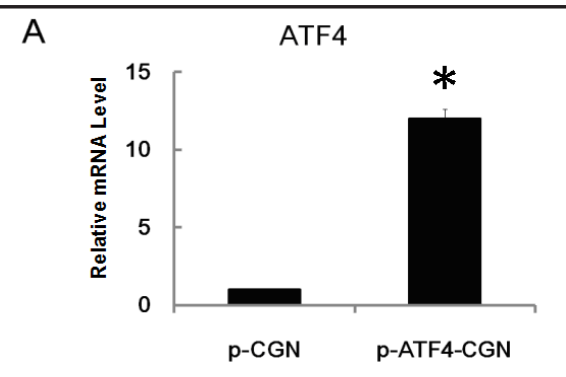

B
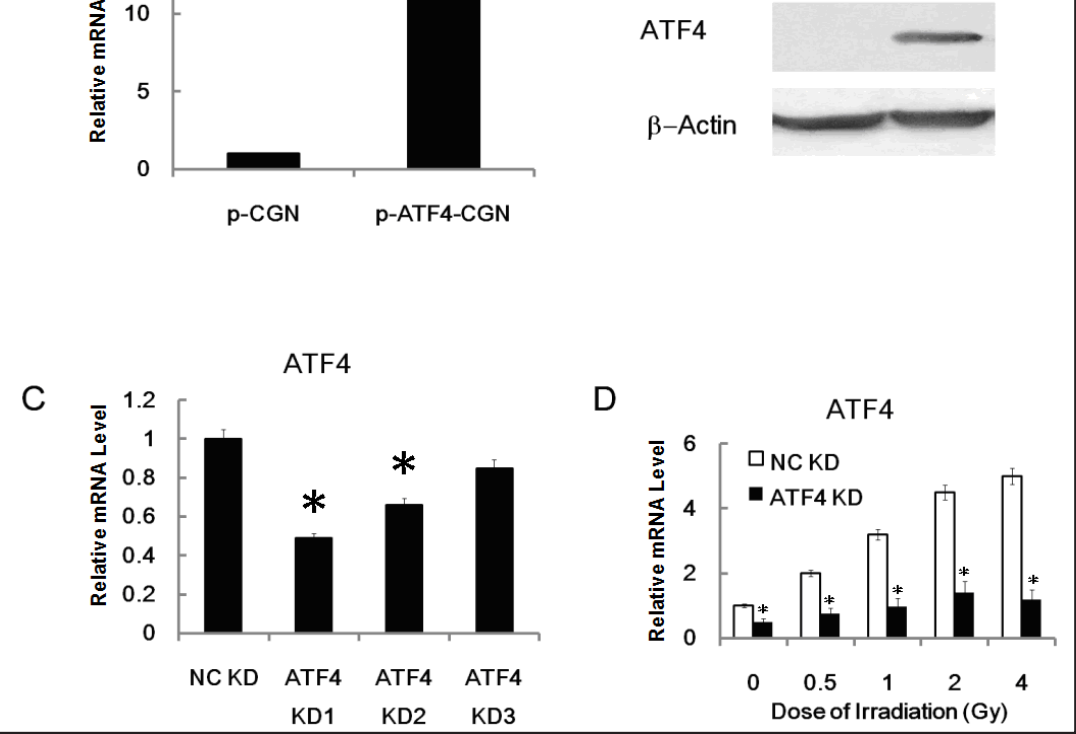

Amaxa Cell Line Nucleofector Kit L, and the relative levels of ATF4 mRNA were analyzed by real-time PCR 24 h later in (Fig. 4C). Under different doses of irradiation, the expression of ATF4 mRNA was detected in the lowest ATF4 mRNA expression level cells (KD1) and it didn't increase in ATF4 knockdown cells (Fig. 4D). Results are representative of 3 independent experiments $* \mathrm{P}<0.05$ vs. control.

ATF4-overexpressing cells than in control cells and gradually increased before peaking after $48 \mathrm{~h}$ (24.2\% in ATF4-overexpressing cells and 8.1\% in control cells) (Fig. 5B). We next examined the effect of ATF4 on the cell cycle and found that in ATF4-overexpressing HEK293 cells, the proportion of cells in G0/G1 phase cells was higher while the proportion in S phase was lower, suggesting that ATF4 induced G0/G1 arrest (Fig. 5C). As shown in Fig. 5D and 5E, over-expressing ATF4 in HEK293 cells suppressed the proportion of BRDU ${ }^{+}$cells and up-regulated the level of ROS in the intracellular compartment. Over-expressing ATF4 promoted radiation-induced cell apoptosis (Fig. 5F), suggesting that ATF4 protected cells from malignant transformation and could be a potential therapeutic target for radiotherapy. These results indicate that a higher level of ATF 4 confers anti-proliferative and pro-apoptotic effects. These data collectively suggest that ATF 4 could promote radiation-induced cell death.

ATF4 knockdown protects cells from IR-induced injury

To confirm the effects of IR-induced ATF4, we used a siRNA strategy. Three sets of ATF4 siRNAs were transfected into ECV304 cells to obtain an ATF4 knockdown system, and $24 \mathrm{~h}$ 
Fig. 5. The effect of ATF4 over expression on cell function. Over-expressed ATF4 could suppress proliferation of HEK293 cells (Fig. 5A) and promote cell apoptosis (Fig. 5B). Over-expression of ATF4 in HEK293 cells caused G1 phase arrest (i.e. it up-regulated the ratio of G1 cells and down-regulated the ratio of $\mathrm{S}$ cells) (Fig. 5C). Over-expression of ATF4 in HEK293 cells suppressed the ratio of $\mathrm{BRDU}^{+}$cells and up-regulated ROS DCFH-DA cells (Fig. 5D\&E). Over-expression of ATF4 promoted apoptosis induced by radiation (Fig. 5F). ${ }^{*} P$ $<0.05$ vs. control.

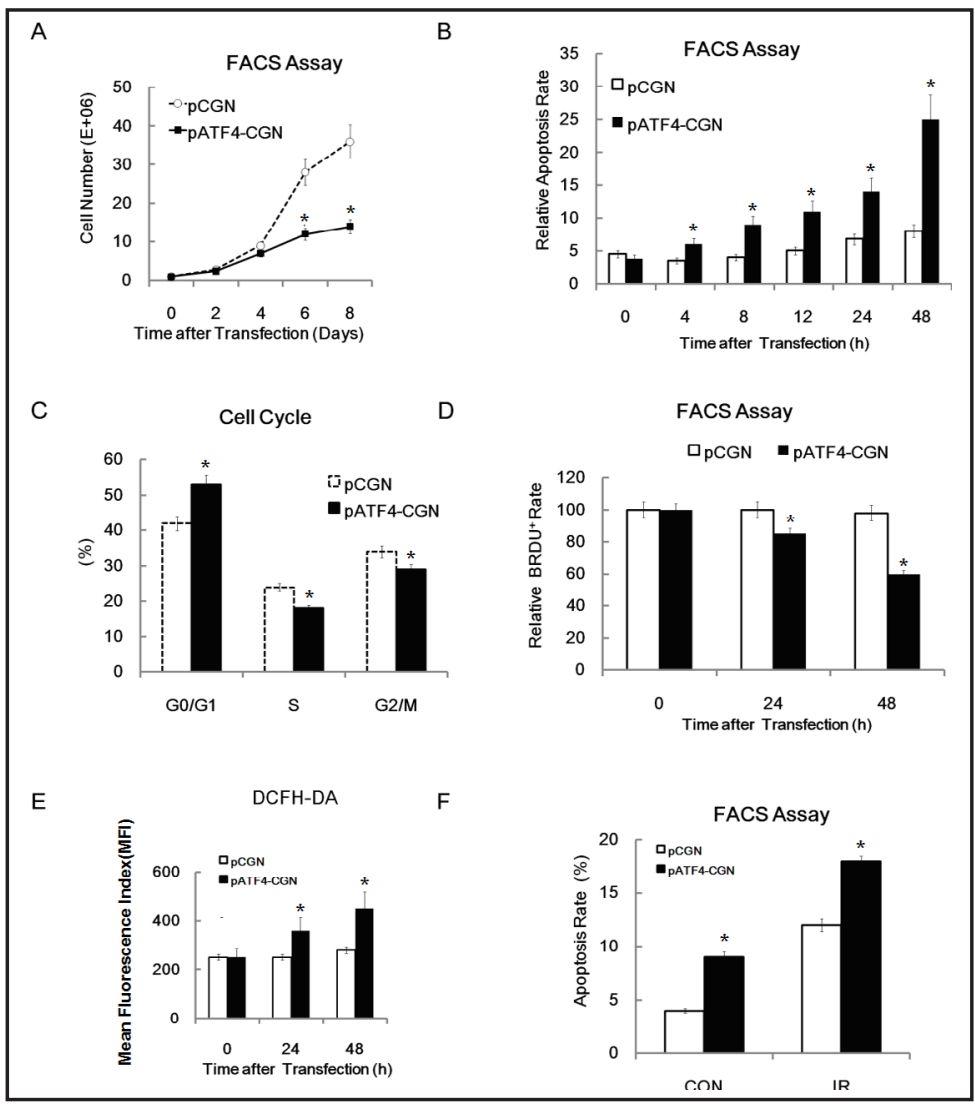

Fig. 6. The effect of ATF4 knockdown on cell function. Knockdown of ATF4 protect ECV304 cells from radiation and promote cell proliferation (Fig. 5A). Knockdown of ATF4 could protect ECV304 cells from apoptosis induced by irradiation (Fig. 5B). Knockdown of ATF4 in ECV304 cells down-regulated the ratio of G1 cells after irradiation (Fig. 5C). Knockdown ATF4 in ECV304 cells could down-regulate ROS DCFH-DA (Fig. 5D). $* P<0.05$ vs. control.
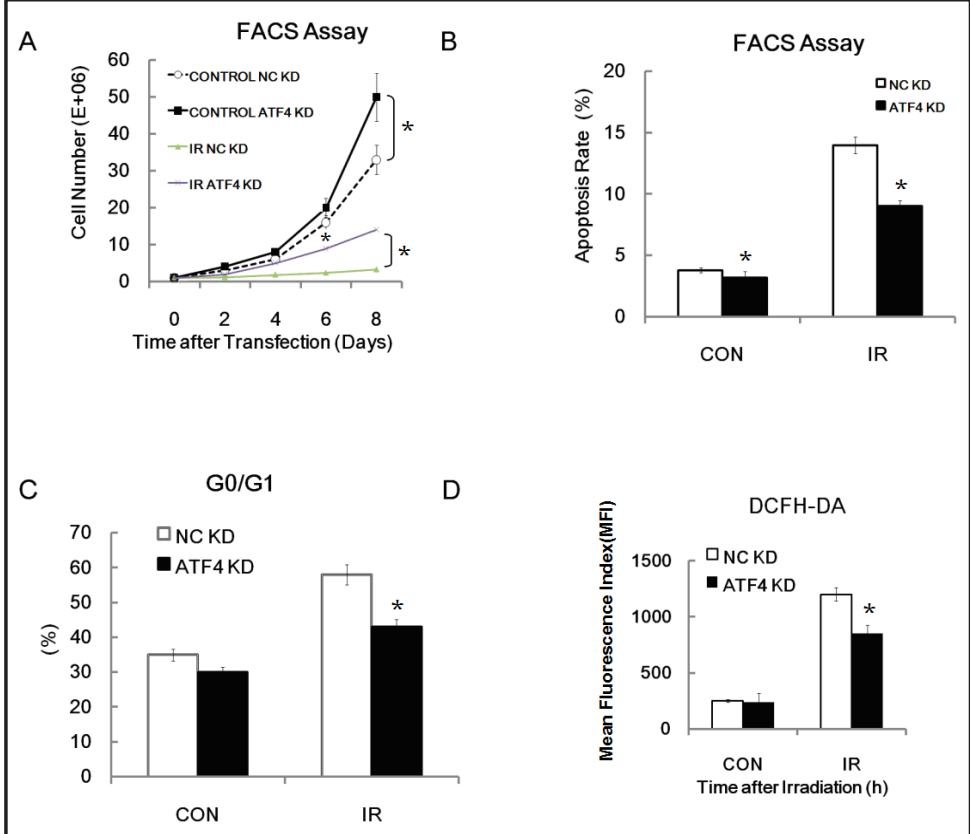

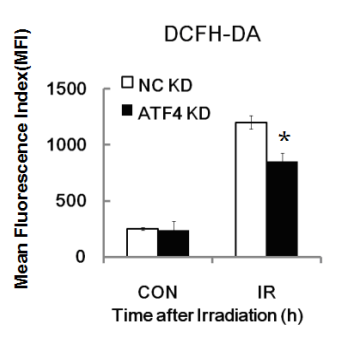

later, the relative levels of ATF4 mRNA were analyzed using real-time PCR. We selected the cells that expressed the lowest levels of ATF4 mRNA (KD1) for follow-up experiments (Fig. 4C \& D). We also determined cell numbers using flow cytometry on different days $(2,4,6$, and 8 days) in ATF4 knockdown ECV304 cells (ATF4 KD1) and ECV304 cells transfected with non-specific control siRNA (NC KD). The total number of ATF4-KD1 ECV304 cells was significantly higher than the number in the control group on the sixth and eighth day. To 

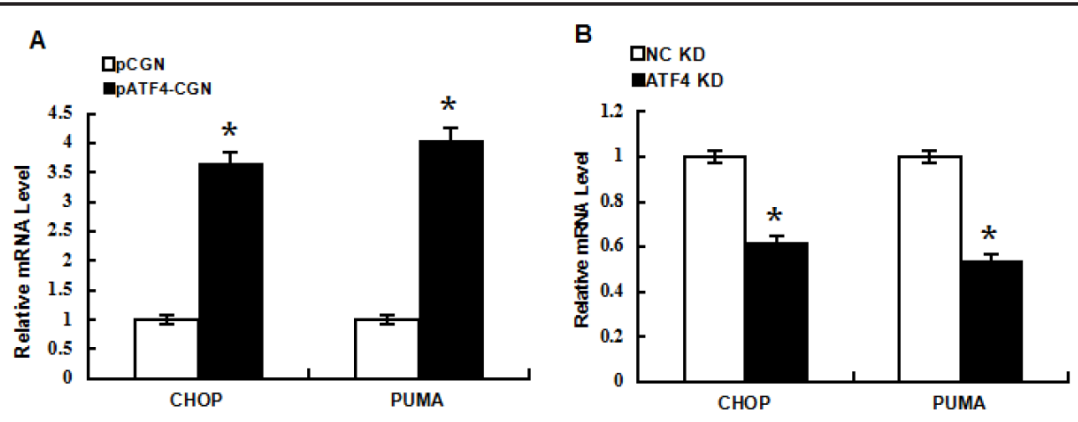

C

D
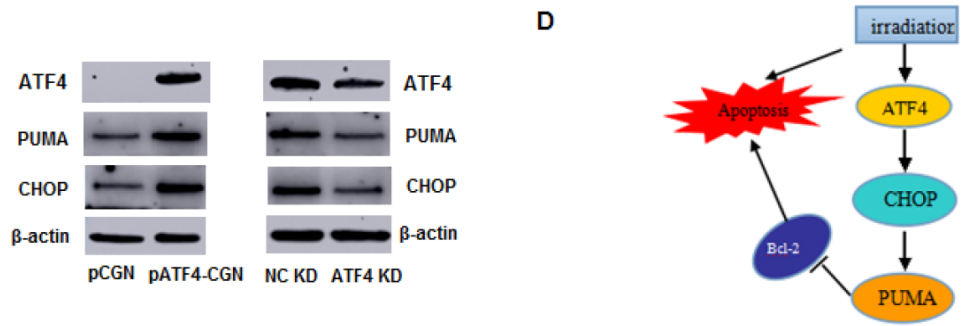

Fig. 7. Chop and puma protein and mRNA expression changed in ATF4 overexpression or knockdown models. The relative expression of chop \& puma mRNA in over-expressed ATF4 HEK 293 cells (Fig. 7A) and in ATF4 KD ECV304 cells (Fig. 7B) were detected using real-time PCR. Immunoblots to measure protein levels in both of the above cell (Fig. 7C). The summary figure of the whole paper (Fig.7D). Results are representative of 3 independent experiments $* P<0.05$ vs. control.

determine whether ATF4 is directly involved in IR-induced cell injury, we also detected the number of ATF4 KD1 and NC KD ECV304 cells after IR with 2 Gy 60Coy and found that the number of ATF4 KD1 cells began to be higher than the number of NC KD cells on the sixth day. The results indicated that ATF4 KD1 cells had less damage than the control group after IR (Fig. 6A). We also detected 2 Gy 60Co $\gamma$ irradiation-induced cell apoptosis after $24 \mathrm{~h}$. The results showed that knocking down ATF4 reduced radiation-induced apoptosis $(14.1 \%$ in ATF4 NC KD cells and 8.5\% in control cells) (Fig. 6B). Therefore, we concluded that ATF4 knockdown adversely affected IR-induced cell damage. An analysis of the cell cycle revealed that when ATF4 was knocked down, the proportion of cells in G0/G1 phase following IR was lower than the proportion in irradiated NC KD ECV304 cells, whereas the proportion of proliferating ATF4 KD1 cells was higher (Fig. 6C). These data showed that ATF4 knockdown enhanced the ability of cells to resist IR. A fluorescence probe, CM-H2DCFDA, was used to determine the levels of ROS that were produced by ECV304 cells. As shown in Fig. 6D, ROS levels were significantly lower in the intracellular compartment in ECV304 cells when ATF4 was knocked down than in control cells.

Under physiological conditions, the balance between ROS generation and ROS scavenging is tightly controlled. However, adaptive physiological redox stress should be differentiated from maladaptive unwanted oxidative damage [25]. Our results showed that ATF4 was associated with intracellular ROS production when cells were exposed to radiation and those higher levels of ROS generation and cell apoptosis were observed in cells with a high level of ATF4 expression than in control cells. Conversely, ATF4 knockdown was associated with less radiation-induced ROS production and apoptosis than were observed in the control group. These results indicate that ATF4 is associated with cellular damage following IR.

The levels of ATF4 target genes are altered in over-expression and knockdown models

To further investigate the mechanisms underlying ATF4 after radiation, we evaluated the expression of genes downstream of ATF4, including C/EBP homologous protein (CHOP) and PUMA, in p-ATF4-CGN HEK293 cells, ATF4-KD1 ECV304 cells and control cells. We found that the mRNA and protein levels of CHOP and PUMA were significantly higher in ATF4 over- 
expressing p-ATF4-CGN HEK293 cells than in control HEK293 cells (Fig. 7A and C). We also determined the mRNA and protein expression levels of CHOP and PUMA and found that they were lower in the knockdown system (ECV304 ATF4-KD1) cells (Fig. 7B, C).

\section{Discussion}

ATF4 plays an important role during many physiological states, including anoxia/ hypoxia, ER stress, amino acid deprivation and oxidative stress. In response to a cell stress signal, ATF4 activates several signaling cascades, resulting in both protective and damaging effects and ultimately leading to cell death [5]. ATF4 expression is triggered by multiple stressors in different cell types. ATF4 induces the transcription of genes involved in redox protection (e.g., HMOX-1 and GCLC), amino acid synthesis (AsnS) and ER stress (e.g., REDD1). ATF4 also up-regulates GADD34, which is involved in apoptosis [9], and genes involved in cell fate decisions (e.g., GADD153/CHOP, ATF3, and CHAC1), which induce cellcycle arrest and trigger apoptosis in cells exposed to intense chronic stress [10, 26-28]. ATF4 results in the initiation of the transcription of the apoptosis-associated CHOP gene and the autophagy-associated Map1lc3b, which selenite links ER stress to apoptosis and autophagy during treatment for leukemia [29]. Zhang et al. showed that histone deacetylase 4 (HDAC4) protects cells from apoptosis-induced ER stress by interacting with ATF4 [30]. ATF4 is also expressed at higher levels in cancer cells than in normal tissue and facilitates tumor growth in xenograft models. ATF4 also induces VEGF and E-selectin, which have been associated with increased metastasis [5]. However, while the phosphorylation of eIF2a and the upregulation of ATF4 enhance cell survival, the hyper-activation of this signaling pathway can lead to permanent cell growth arrest or cytotoxicity as a result of the prolonged inhibition of protein synthesis [15]. An increasing amount of preclinical and clinical data suggests that ATF4 plays important roles in cancer progression and resistance to therapy. These features suggest that ATF4 is an attractive target for anticancer therapeutics. However, our results suggest a different conclusion [5].

In this paper, we report that ATF4 expression is increased in response to radiation in both AHH1 cells and primary murine spleen cells in both a dose- and time-dependent manner. Previous studies have shown that ATF4 is regulated at the post-transcriptional level [31]. In our study, we found that ATF4 mRNA was induced in many human cell lines by ${ }^{60} \mathrm{Co} \gamma$ radiation. ATF 4 expression was markedly suppressed in ATF4 knockdown ECV304 cells. We found that down-regulating ATF4 protected ECV304 cells from radiation and promoted cell proliferation because G1 cell arrest was reduced after ${ }^{60} \mathrm{Co} \gamma$ radiation. These data suggest that the loss of ATF4 enhanced cell survival. We also found that over-expressing ATF4 blocked proliferation in HEK293 and promoted radiation-induced apoptosis. It also significantly up-regulated the proportion of HEK293 cells in G1 phase, suggesting that ATF4 plays a role in radiation-induced apoptosis. We also observed that IR up-regulated ATF4 and the expression of its target genes, CHOP and PUMA. IR has been shown to activate the proapoptotic molecule Puma by activating ATF4 and CHOP. ATF4 over-expressing p-ATF4-CGN HEK293 cells express high levels of CHOP/PUMA mRNA and protein. CHOP/PUMA mRNA and protein expression levels were lower in ECV304 ATF4-KD1 cells, indicating that ATF4 knockdown reduced the expression of CHOP/PUMA at the mRNA and protein expression levels. These results demonstrate that ATF4 is associated with IR-induced cell damage. Promoting the expression of the ATF4 target gene CHOP and its downstream pro-apoptotic target protein PUMA caused cells enter apoptosis (Fig. 7D).

To the best of our knowledge, the present study is the first to demonstrate that IR upregulates the expression of ATF4, leading to a significant increase in radiation-induced cell apoptosis. We hypothesize that ATF4 protects cells from malignant transformation and that ATF4 is therefore a potential therapeutic target for radiotherapy. However, additional studies are required to determine the mechanism underlying this activity.

\section{KARGER}




\section{Cellular Physiology Cell Physiol Biochem 2017;41:784-794 \begin{tabular}{l|l|l}
\hline DOI: 10.1159/000458742 & (C) 2017 S. Karger AG, Basel
\end{tabular}

Zong et al.: Up-Regulated ATF4 Increases Cell Apoptosis in Response to IR

\section{Acknowledgements}

This work was supported by the Major Project of National Science and Technology (2014ZX09J14106-06C, 13CXZ005), the National Natural Science Foundation of China (81473291, 81402651).

\section{Disclosure Statement}

The authors declare no conflict of interest.

\section{References}

1 Harding HP, Zhang Y, Zeng H, Novoa I, Lu PD, Calfon M, Sadri N, Yun C, Popko B, Paules R, Stojdl DF, Bell JC, Hettmann T, Leiden JM, Ron D: An integrated stress response regulates amino acid metabolism and resistance to oxidative stress. Mol Cell 2003;11:619-633.

2 Rutkowski DT, Kaufman RJ: All roads lead to ATF4. Dev Cell 2003;4:442-444.

3 Tanabe M, Izumi H, Ise T, Higuchi S, Yamori T, Yasumoto K, Kohno K: Activating transcription factor 4 increases the cisplatin resistance of human cancer cell lines. Cancer Res 2003;63:8592-8595.

- Z Zhang Z, Yin J, Zhang C, Liang N, Bai N, Chang A, Liu Y, Li Z, Tan X, Li N, Lv D, Xiang R, Tian Y, Liu C: Activating transcription factor 4 increases chemotherapeutics resistance of human hepatocellular carcinoma. Cancer Biol Ther 2012;13:435-442.

-5 Ameri K, Harris AL: Lactivating transcription factor 4. Int J Biochem Cell Biol 2008;40:14-21.

6 Horiguchi M, Koyanagi S, Okamoto A, Suzuki SO, Matsunaga N, Ohdo S: Stress-regulated transcription factor ATF4 promotes neoplastic transformation by suppressing expression of the INK4a/ARF cell senescence factors. Cancer Res 2012;72:395-401.

7 Singleton DC, Harris AL: Targeting the ATF4 pathway in cancer therapy. Expert Opin Ther Targets 2012;16:1189-1202.

8 Igarashi T, Izumi H, Uchiumi T, Nishio K, Arao T, Tanabe M, Uramoto H, Sugio K, Yasumoto K, Sasaguri Y, Wang KY, Otsuji Y, Kohno K: Clock and ATF4 transcription system regulates drug resistance in human cancer cell lines. Oncogene 2007;26:4749-4760.

9 Li S, Guo L, Qian P, Zhao Y, Liu A, Ji F, Chen L, Wu X, Qian G. Lipopolysaccharide Induces Autophagic Cell Death through the PERK-Dependent Branch of the Unfolded Protein Response in Human Alveolar Epithelial A549 Cells. Cell Physiol Biochem 2015;36:2403-2417.

10 Magne L, Blanc E, Legrand B, Lucas D, Barouki R, Rouach H, Garlatti M: ATF4 and the integrated stress response are induced by ethanol and cytochrome P450 2E1 in human hepatocytes. J Hepatol 2011;54:729737.

11 Mungrue IN, Pagnon J, Kohannim O, Gargalovic PS, Lusis AJ: CHAC1/MGC4504 is a novel proapoptotic component of the unfolded protein response, downstream of the ATF4-ATF3-CHOP Cascade. J Immunol 2009;182:466-476.

12 Wali JA, Rondas D, McKenzie MD, Zhao Y, Elkerbout L, Fynch S, Gurzov EN, Akira S, Mathieu C, Kay TW, Overbergh L, Strasser A, Thomas HE:The proapoptotic BH3-only proteins Bim and Puma are downstream of endoplasmic reticulum and mitochondrial oxidative stress in pancreatic islets in response to glucotoxicity. Cell Death Dis DOI:10.1038/cddis.2014.88.

13 Puthalakath H, O’Reilly LA, Gunn P, Lee L, Kelly PN, Huntington ND, Hughes PD, Michalak EM, McKimmBreschkin J, Motoyama N, Gotoh T, Akira S, Bouillet P, Strasser A: ER stress triggers apoptosis by activating BH3-only protein Bim. Cell 2007;129:1337-1349.

14 Galehdar Z, Swan P, Fuerth B, Callaghan SM, Park DS, Cregan SP: Neuronal apoptosis induced by endoplasmic reticulum stress is regulated by ATF4-CHOP-mediated induction of the Bcl-2 homology 3-only member PUMA. J Neurosci 2010;30:16938-16948.

15 Kim EJ, Lee YJ, Kang S, Lim YB: Ionizing radiation activates PERK/eIF2 $\alpha /$ ATF4 signaling via ER stressindependent pathway in human vascular endothelial cells. Int J Radiat Biol 2014;90:306-312. 


\section{Cellular Physiology Cell Physiol Biochem 2017;41:784-794 \begin{tabular}{l|l|}
\hline DOI: 10.1159/000458742 & O 2017 S. Karger AG, Basel \\
\hline
\end{tabular}

Zong et al.: Up-Regulated ATF4 Increases Cell Apoptosis in Response to IR

16 Xu RX, Liu RY, Wu CM, Zhao YS, Li Y, Yao YQ Xu YH. DNA damage-induced NF-кB activation in human glioblastoma cells promotes miR-181b expression and cell proliferation. Cell Physiol Biochem 2015;35:913-925.

17 Daly MJ, Gaidamakova EK, Matrosova VY, Vasilenko A, Zhai M, Leapman RD, Lai B, Ravel B, Li SM, Kemner KM, Fredrickson JK: Protein oxidation implicated as the primary determinant of bacterial radio resistance. PLOS Biol DIO:10.1371/journal.pbio.0050092.

18 Guida MS, Abd El-Aal A, Kafafy Y, Salama SF, Badr BM, Badr G. Thymoquinone rescues T lymphocytes from Gamma irradiation-induced apoptosis and exhaustion by modulating pro-inflammatory cytokine levels and PD-1, Bax, and Bcl-2 Signaling. Cell Physiol Biochem 2016;38:786-800.

19 Wong MK, Takei Y: Angiotensin AT2 receptor activates the cyclic-AMP signaling pathway in eel. Mol Cell Endocrinol 2013;365:292-302.

20 Liu C, Li B, Cheng Y, Lin J, Hao J, Zhang S, Mitchel RE, Sun D, Ni J, Zhao L, Gao F, Cai J: Mir-21 plays an important role in radiation induced carcinogenesis in BALB/c mice by directly targeting the tumor suppressor gene big-h3. Int J Biol Sci 2011;7:347-363.

21 Liu C, Zhou C, Gao F, Cai S, Zhang C, Zhao L, Zhao F, Cao F, Lin J, Yang Y, Ni J, Jia J, Wu W, Zhou L, Cui J, Zhang W, Li B, Cai J: Mir-34a in age and tissue related radio-sensitivity and serum mir-34a as a novel indicator of radiation injury. Int J Biol Sci 2011;7:221-233.

22 Zhang S, Hao J, Xie F, Hu X, Liu C, Tong J, Zhou J, Wu J, Shao C: Downregulation of mir-132 by promoter methylation contributes to pancreatic cancer development. Carcinogenesis 2011;32:1183-1189.

23 Zhang Y, Wang YG, Zhang Q Liu XJ, Liu X, Jiao L, Zhu W, Zhang ZH, Zhao XL, He C: Interaction of mint2 with trka is involved in regulation of nerve growth factor-induced neurite outgrowth. J Biol Chem 2009;284:12469-12479.

24 Su S, Li Y, Luo Y, Sheng Y, Su Y, Padia RN, Pan ZK, Dong Z, Huang S: Proteinase-activated receptor 2 expression in breast cancer and its role in breast cancer cell migration. Oncogene 2009;28:3047-3057.

25 Zorov DB, Juhaszova M, Sollott SJ:Mitochondrial reactive oxygen species (ROS) and ROS-Induced ROS Release. Physiol Rev 2014;94:909-950.

26 Cazanave SC, Elmi NA, Akazawa Y, Bronk SF, Mott JL, Gores GJ: CHOP and AP-1 cooperatively mediate PUMA expression during lipoapoptosis. Am J Physiol Gastrointest Liver Physiol 2010;299:G236-G243.

27 Szegezdi E, Logue SE, Gorman AM, Samali A: Mediators of endoplasmic reticulum stress-induced apoptosis. EMBO Rep 2006; 7:880-885.

28 Jiang HY, Wek SA, McGrath BC, Lu D, Hai T, Harding HP, Wang X, Ron D, Cavener DR, Wek RC: Activating transcription factor 3 is integral to the eukaryotic initiation factor 2 kinase stress response. Mol Cell Biol 2004;24:1365-1377.

29 Jiang Q, Li F, Shi K, Wu P, An J, Yang Y, Xu C: ATF4 activation by the p38MAPK-eIF4E axis mediates apoptosis and autophagy induced by selenite in Jurkat cells. FEBS Lett 2013;587:2420-2429.

-30 Zhang PF, Sun Q, Zhao CY, Ling SK, Li Q Chang YZ, Li YX: HDAC4 protects cells from ER stress induced apoptosis through interaction with ATF4. Cell Signal 2014;26:556-563.

- 31 Zhang CY, Bai N, Chang AT, Zhang ZH, Yin J, Shen WZ,Tian YP, Xiang R, Liu C: ATF4 is directly recruited by TLR4 signaling and positively regulates TLR4-trigged cytokine production in human monocytes. Cell Mol Immunol 2013;10:84-94. 